\section{Autobiographie}

Heinz Stefan Herzka Unterwegs im Zwischen.

Eine Autobiographie

Frauenfeld, Stuttgart, Wien: Huber; 2007

468 Seiten, zahlreiche Abbildungen, gebunden. Fr. 48.-

ISBN: 978-3-7193-1442-2

Der Buchtitel ist Programm. Wir erfahren, wo und wie Heinz Stefan Herzka (HSH) unterwegs war. Die Umstände seiner ersten «Reise» von Wien in die Schweiz als verfolgter Jude haben sein Leben geprägt, belastet. Ein Teil des Zwischen ist damit auch erklärt. Der andere Teil des Zwischen liegt in seiner intellektuellen Neugier. Er gab sich nicht mit einem Standpunkt, einem Fach zufrieden. Die Flucht wurde ihm und seiner Familie aufgezwungen, die intellektuelle Neugier gehört zu seinem Wesen. Die Flucht ermöglichte das nackte Überleben, die intellektuelle Neugier bescherte ihm Einsichten, Freundschaften, Bewunderung, nicht nur der Studenten. Für beides, Flucht und intellektuelle Neugier, bezahlte er aber auch einen hohen Preis. Das wird immer wieder spürbar.

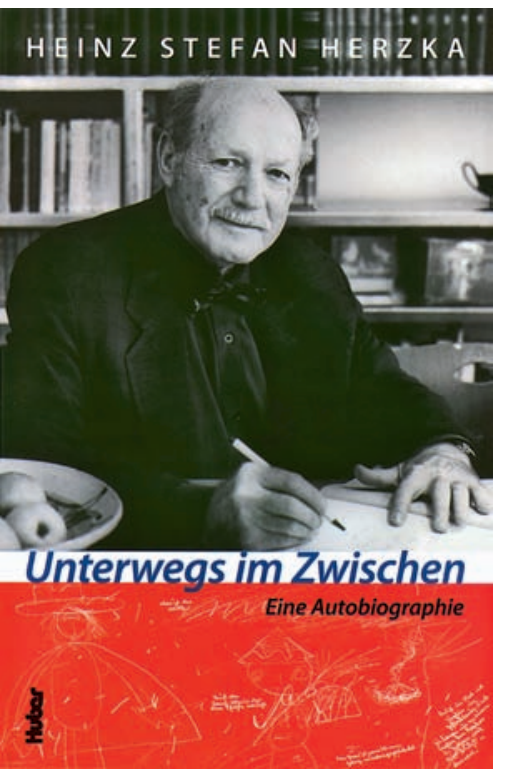

HSH räumt der Schilderung seiner familiären Wurzeln breiten Raum ein. Das ist auch darum so spannend, weil er über eindrückliche Dokumente, die von Verwandten verfasst wurden, verfügt. So kann $\mathrm{HSH}$ auch nachvollziehbar beschreiben, wie seine Eltern sich bereits in den 20er Jahren aus ihrer familiären Tradition emanzipierten, da sie vom Wertewandel der adoleszenten Generation nach dem Ersten Weltkrieg geprägt waren. In diesem «68er» Geist wurde HSH, geboren 1935, erzogen. Von der Mutter gibt es viele von ihr verfasste Artikel und Notizen. Eine Studentin von HSH schrieb gar ihre Doktorarbeit über diese Mutter, Psychologin, Mitarbeiterin von Alfred Adler. Ihre berufliche Karriere wurde durch die Flucht beendet, zerstört. Der plötzliche Kindstod der Nachzüglertochter 1946 brachte nochmals unermessliches Leid in diese Emigrantenfamilie. Der Vater, studierter Wirtschaftswissenschaftler, in seiner beruflichen Entwicklung genauso behindert wie seine Frau, erscheint in und vor allem zwischen den Zeilen von HSH als wirklich eindrückliche Person. Er emigrierte nach der Heirat von HSH nach Israel. Die Mutter starb 1954, der Vater 1977.

Interessant zu lesen ist die berufliche Entwicklung von HSH, wie er mit Pädiatrie, Pädagogik und Philosophie seinen Weg in der Kinder- und Jugendpsychiatrie fand. «Die Einführung der Dialogik in die Medizin und Psychologie bleibt für mich das nachhaltigste Ergebnis meiner wissenschaftlichen und publizistischen Arbeit» (S. 288).

HSH zitiert sich aus dem Jahr 1992: «Das dialogische Prinzip besagt, dass zwei Gedanken, die niemand gleichzeitig denken kann, oder zwei Begriffe, die sich gegenseitig ausschliessen und je einen Bereich für sich bezeichnen, gleichzeitig (das heißt nicht nacheinander) und gleichwertig (d.h. ohne Überlegenheitsanspruch und Unterordnung) gemeinsam ein Ganzes ausmachen» (S 289).

«Unterwegs im Zwischen» ist eine interessante Entwicklungsgeschichte. Das macht das Buch lesenswert. HSH hat sich über Jahrzehnte durch seine Vorlesungen, seine Referate und Publikationen grosse Verdienste erworben um die Wahrnehmung der Bedürfnisse der Kinder und Jugendlichen, besonders der benachteiligten. Er hat sich auch grosse Verdienste erworben um die interdisziplinäre Zusammenarbeit von Pädagogen und Therapeuten. Hier ist er in seiner Autobiographie zu bescheiden, gibt dem zu wenig Raum.

HSH hat beruflich nicht alle Ziele erreicht, die er sich gesteckt hat. Die Abrechung mit seinen Widersachern ist in der Autobiographie etwas zu umfangreich geraten.

Speziell berührt hat mich die Beschreibung eines Besuches von HSH bei Jaspers 1967. In der Autobiographie ist ein Auszug aus den Notizen von HSH unmittelbar nach dem Besuch enthalten. Was mich hier besonders faszinierte, war die Sprache, in der dieser doch sehr sonderbare Besuch beim alten Herrn geschildert wird (S. 259ff.).

Schön, dass das Buch mit der Beschreibung des zweiten grossen Lebensthemas neben der Dialogik endet: den Schalmeien. HSH schildert seine Reisen rund um die Welt auf der Suche nach diesen Instrumenten, wie er die Geschichte dieses Instrumentes studierte, eine umfangreiche Sammlung anlegte und diese nun in Frankreich in einem Museum unterbringen konnte. Mit diesem Thema war und ist das Unterwegssein für HSH erfreulich und bereichernd. Wenn er musiziert, ist er, wie aus

seinen Schilderungen zu entnehmen ist, nicht mehr zwischen, sondern mittendrin.

Wilhelm Felder, Bern

\title{
Expérience de médecin
}

\section{Christian Müller}

Miniatures psychiatriques -

Témoignages d'un médecin au travail

Genève: Labor et Fides; 2007

Fr. 28.-

ISBN: 978-2-8309-1237-1

Christian Müller est une figure de la psychiatrie suisse, de la Faculté de médecine de Lausanne et de la santé publique vaudoise. Il a dirigé de 1961 à 1987 l'Hôpital psychiatrique universitaire de Cery. Après avoir été son étu-

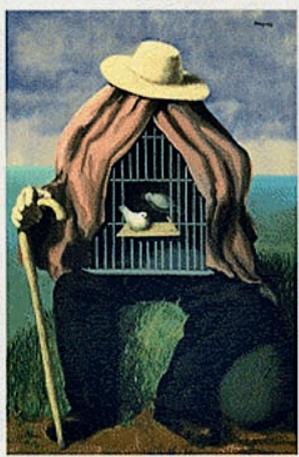

Christian Müller

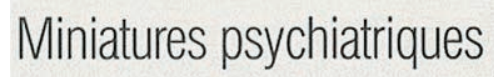

Témoignages d'un médecin au travail

\section{LAROB ET FIDES}

diant peu après son arrivée, j'ai eu l'occasion de le côtoyer, en tant que médecin cantonal adjoint, dans la dernière décennie de son mandat. C'est dire mon intérêt à lire ses «Miniatures psychiatriques», quarantaine de vignettes cliniques et sociales sur son expérience de médecin - et, dans plusieurs des cas décrits, de confrère.

Dans mon souvenir, il était un confrère et un maître réfléchi, calme, expliquant patiemment, pesant ses mots. Patron à qui rien de ce qui concernait son établissement ne saurait être étranger, attentif aux aspects domestiques et hôteliers comme à ce qui concernait la thérapeutique et l'enseignement.

Cela apparaît bien dans ces courts récits, agréables à lire, présentés dans des chapitres portant chacun sur une décennie, des années 50 alors que l'auteur était médecin assistant jusqu'aux années 80. Quelques facettes de la psychiatrie 
avant les psychotropes, très institutionnelle, où l'hôpital devenait le domicile d'une proportion notable des patients. Communauté avec sa vie propre; le déroulement de l'année était marqué par des moments très sociaux/ sociables de fête (la mutation qui, pour donner à l'hôpital psychiatrique un caractère d'établissement de soins aigus, entraîna le déplacement de résidents de très longue date n'a pas été sans déchirements). Puis l'ouverture, avec des interactions et mouvements nettement plus fréquents entre dedans et dehors. Description des circonstances, parfois des vicissitudes petites et grandes, de la vie de l'institution.

«Miniatures psychiatriques» est un livre par un médecin mais pas un livre médical; accessible à tout public intéressé par l'évolution de notre société et du système de soins. Racontée avec un recul correspondant bien à l'auteur. Et pour finir le départ: «Est-ce que j'ai eu de la peine en vidant les armoires, en décrochant les tableaux? Oui. Pourtant je m'étais préparé, je m'étais habitué à l'idée de prendre ma retraite.» Et peu après, alors qu'il passe par l'hôpital, une infirmière nouvelle lui demande s'il est le mari d'une patiente ... Sic transit ...

Jean Martin, Echandens

\section{Sterbebegleitung}

\section{Klara Obermüller \\ Weder Tag noch Stunde. \\ Nachdenken über Sterben und Tod}

Frauenfeld: Huber; 2006

156 Seiten

Fr. 29.80

ISBN: 978-3-7193-1445-3

«Ein wichtiges, ausserordentliches und eminent nützliches Buch, das keineswegs nur von Kranken und ihren Angehörigen, sondern auch von Gesunden und eigentlich von jedermann gelesen werden sollte!» - so könnte man den Gesamteindruck zusammenfassen, wenn man das Buch von Klara Obermüller, der Witwe des 1978 an Krebs verstorbenen Schriftstellers Walter Matthias Diggelmann, aus der Hand legt. Sie geht von diesem einschneidenden $\mathrm{Er}$ lebnis aus und berichtet über weitere Menschen, die sie bis zum Tod begleitet hat. Mehr noch als mit dem Erleben der Sterbenden, von denen uns nach ihrer Ansicht ein letztlich kaum zu überbrückender Graben trennt, beschäftigt sie sich dabei auch mit dem, was deren Nächsten und Angehörigen widerfährt - mit ihrer Trauer, ihrer Einsamkeit und Ohnmacht, ihren Schuld- und Überforderungsgefühlen bis hin zu manchmal auftauchenden Gefühlen von Feindseligkeit. Das Buch ist, so merkwürdig dies bei einem solchen Thema auch scheinen mag, enorm spannend geschrieben. Die Sprache ist dicht, klar, eindringlich und, so wil mir scheinen, ohne jeden Abstrich authentisch; man möchte immer nur zitieren ... Zum Beispiel: «Einen Menschen sterben sehen ist unendlich schwer: einfach da sein, nichts mehr tun können, den andern gehen lassen. Wohin genau, wer weiss es?» oder «Meiner Ansicht nach ist es wichtig, dass der Patient Bescheid weiss über seinen Zustand und dass man die letzte Zeit, die einem noch bleibt, nicht mit Lügen zubringt.» Allein die Kapitelüberschriften sprechen Bände: «Der lange Weg» - «Zu Hause sterben» - «Die Wahrheit ist dem Menschen zumutbar» - «Am Ende der ärztlichen Kunst» - «Ars moriendi» - «Therapie der Ehrlichkeit» - «Sprache suchen für das, was uns stumm macht» - «Es gibt nicht nur die Krankheit, es gibt auch das Leben» - «Der Tod ist gross. Wir sind die Seinen». Rilke, Camus, Celan, Elisabeth Kübler-Ross, Peter Noll und viele andere, die sich über Tod und Sterben Gedanken gemacht haben, kommen ebenfalls zu Wort - ohne jedes literarische Getue. Wohltuend ist auch, dass die Autorin den Tod nicht mystifiziert, nicht «auf die andere Seite» schauen will, sondern sich vorab mit dem Leben beschäftigt, zu dem der Tod unabdinglich gehört. Trotz - oder gerade wegen dieser Zurückhaltung fehlt indes eine «spirituelle Dimension» keineswegs. Klara Obermüller geht keinem Problem aus dem Weg, nicht der Verdrängung und gleichzeitigen Banalisierung des Todes in den Medien, nicht seiner Vertechnisierung in der heutigen Spitzenmedizin und auch nicht seiner Industrialisierung in den Todeslagern des Zweiten Weltkrieges. Sie plädiert für das Sterben zu Hause, für den langen gemeinsamen Weg bis zur Annahme des Todes, ohne die Notwendigkeit von Zeiten der (z.B. ärztlichen) Auflehnung zu leugnen. «Ich begriff, dass wir etwas verloren haben, als wir den Tod aus unserem Leben verbannten. Meine eigenen Erfahrungen mit dem Sterben naher Menschen haben mich gelehrt, wie hilfreich ein Abschied am Sterbebett, wie heilsam ein gemeinsames Trauern, ein Reden und Erinnern und Geschichtenerzählen für diejenigen sein können, die weiterleben müssen» - Noch einmal: ein Buch, das ich jedem Betroffenen empfehlen möchte - und wer wäre nicht Betroffene(r), früher oder später?

Luc Ciompi, Belmont-sur-Lausanne

\section{Versicherungsmedizin}

Gabriela Riemer-Kafka, Universität Luzern (Hrsg.) Versicherungsmedizinische Gutachten

Ein interdisziplinärer juristisch-medizinischer Leitfaden

Bern: Stämpfli / Basel: EMH; 2007

64 Seiten, Taschenbuch.

Fr. 28.

ISBN: 978-3-03754-003-9
Wer sich als Arzt auf dieses Glatteis begeben will, ist gut beraten, wenn er vorher diesen Leitfaden aufmerksam liest.

Interdisziplinär. Dabei denkt man sich ein Gespräch der verschiedenen Kontrahenten auf Augenhöhe. Bei der Lektüre wird man jedoch bald eines Besseren belehrt: Die Disziplin hat hier eine etwas andere Bedeutung: Die Disziplinierung des medizinischen Gutachters durch die «Rechtsanwender», d.h. die Versicherungen und ihre Organe, und, hierarchisch diesen noch übergeordnet, die Juristen.

Das manifestiert sich bereits in der Sprache: Die Sprache war immer schon jene des Herrn, nicht jene des Dieners und diente der Disziplinierung der Untergeordneten. So auch in diesem Leitfaden. Ein Beispiel: «Der medizinische Gutachter hat [...] auf der Basis der an ihn gestellten Sachfragen für die von der rechtsanwendenden Stelle zu vollziehende Subsumption [ein paar Zeilen weiter unten ist von Subsumtion die Rede; Anm. des Autors] unter den Tatbestand aufzuarbeiten und darin darzulegen. Darin erschöpft sich gegenüber dem Auftraggeber seine Aufgabe.» Alles klar? Noch Fragen?

So wird in verschiedenen Kapiteln genau vorgeschrieben, was der Gutachter zu tun, zu beachten, genau zu befolgen und was er anderseits zu lassen, was er sich nicht anzumassen und wovor er sich zu hüten habe.

Dass medizinische Fachsprache, im Gegensatz $\mathrm{zu}$ ausufernder juristischer, im vorliegenden Heft praktisch nicht vorkommt, gereicht ihm zur Ehre und zum Vorteil, hat aber auch den einfachen Grund darin, dass «weder Arzt noch Jurist befähigt sind, sich im anderen Fachgebiet kompetent zu bewegen» (dieser Satz ist so wichtig, dass er im vorliegenden Heft gleich zweimal erscheint). Da aber der Jurist (in letzter Instanz der Richter) Auftraggeber ist, und er die Sprache der Medizin nicht versteht, muss die Sache in Laiensprache dargestellt werden. Das leuchtet ohne weiteres ein. Und dass Juristen offenbar auch mit verhältnismässig einfachen medizinischen Sachverhalten Mühe haben, zeigt etwa folgendes Beispiel: «Ebenso gehört es zur Aufgabe des Gutachtens, nicht objektivierbare Beschwerden als solche $\mathrm{zu}$ kennzeichnen und auf die Gründe der nicht möglichen Belegbarkeit und allfälligen Quantifizierbarkeit hinzuweisen», wie wenn Subjektives, wie etwa (definitionsgemäss) Beschwerden, auch einmal objektivierbar wäre. Die Asymmetrie des Auftragsverhältnisses zieht sich aber auch weiter durch das ganze Büchlein: «dass ein Arzt in einem Anstellungsverhältnis zum Versicherungsträger steht, lässt nicht schon auf mangelnde Objektivität und Befangenheit schliessen», hingegen «soll das Gericht der Erfahrungstatsache Rechnung tragen, dass Hausärzte mitunter im Hinblick auf ihre auftragsrechtliche Vertrauensstellung in Zweifelsfällen eher zugunsten ihrer Patienten aussagen (BEG 125 V 353 Erw. 3b/cc)». 
Versicherungsmedizinische Gutachten waren immer schon eine Krux und ein Ärgernis. Und sie werden es bleiben. Was ist ihr Zweck? Gerechtigkeit gibt's wohl erst im Himmel. Auf Erden muss das Recht herrschen, und das ist recht so und die Aufgabe der Juristen. Und da ihnen der medizinische Sachverstand fehlt, sind sie auf die Hilfe der Ärzte angewiesen. Diese aber sind, aus ihrer Herkunft und Motivation, eher an einer wie auch immer gedachten Gerechtigkeit interessiert, durchaus im Gegensatz zur anderen Seite. So sind sie in einem Zwiespalt. Gutachten sind notorisch bei den Ärzten unbeliebt. Man erledigt sie als Pflichtübung, weil man einsieht, dass es sie braucht. Etwa so, wie wir auch Militärdienst geleistet haben. Ungern, aber trotzdem.

Manchen Ärzten dient die Gutachtertätigkeit auch zum Broterwerb, der anderweitig oft nicht mehr gesichert erscheint. Sie leisten einen wichtigen Beitrag zum Funktionieren unseres Gesundheitswesens. Die Disziplinierung durch die Auftraggeber, durch Versicherungen und Juristen, ist nicht zu vermeiden. Es ist gut, wenn sich die Ärzte über diese Einbahnstrasse bzw. Asymmetrie genau im klaren sind. Deshalb sei ihnen empfohlen, diese preiswerte, knappe Schrift zu erstehen und genau durchzulesen.

Was für mich persönlich in langer Gutachtertätigkeit immer wieder eine sportliche Herausforderung war: Mit dem «Ermessensspielraum», der dem Arzt aufgrund seines Fachwissens auch vom Auftraggeber zugestanden wird, ein wenig zu «spielen» und der «Gerechtigkeit» dort, wo ich sie sah, durch geschickte Formulierung auch zu ihrem «Recht» zu verhelfen. Durchaus nicht immer erfolglos.

Eine Brücke möchte das Heft schlagen? Da steigen mir Zweifel auf. Dass der Graben viel zu breit und zu tief ist, habe ich selbst immer wieder und zuletzt recht eindeutig an einem interdisziplinären Seminar der Deutschen Anwalt Akademie $\mathrm{GmbH}$ erfahren.
Da medizinische Gutachten für einen geregelten Betrieb aber unerlässlich sind, erscheinen bei dieser Lage der Dinge genaue Richtlinien umso wichtiger. Solche liefert dieses Heft in aller wünschbaren Klarheit. Es sollte deshalb Pflichtlektüre für beide Seiten sein.

Alfred M. Debrunner, Uitikon / Zürich

\section{Kommunikation}

\section{Karsten Bredemeier}

\section{Der Rhetorik-Code}

Zürich: Orell Fuessli; 2007

104 Seiten, gebunden.

Fr. 29.80

ISBN: 978-3-280-05241-9

Der bekannte Kommunikationswissenschaftler Karsten Bredemeier präsentiert in seinem aktuellsten Werk basierend auf soziopsychologischen Grundlagen und neuesten Kenntnissen der Informationsverarbeitung den sogenannten «Rhetorik-Code». In zehn Grundregeln wird kurz und prägnant die Basis moderner angewandter Kommunikationswissenschaft für den Alltag dargelegt und mit ergebnissichernden Verhaltensregeln untermauert.

Das über 100 Seiten lange Buch zeigt in aller Deutlichkeit auf, dass gute und damit wirkungsvolle Kommunikation für Führungskräfte heute keinen Zufall mehr darstellt, sondern das Ergebnis harter Arbeit und Schulung ist. Dass solche «soft skills» schon längst Eingang in das Krankenhausmanagement gehalten haben, wissen wir nicht zuletzt auch aufgrund des konsequenten Medientrainings von (Chef-)Ärzten in deutschen Privatkliniken. Aber selbst in der Hochschuldidaktik wird heute der Rhetorik grosse Bedeutung zugemessen, da die Beherrschung des gesproche- nen Wortes eine der wichtigsten Voraussetzungen für eine erfolgreiche Lehre darstellt. Dem Autor gelingt es im vorliegenden Werk in eindrücklicher Art und Weise, die von ihm propagierten Kommunikationsregeln konsequent und damit vorbildhaft umzusetzen. Damit liefert der Autor den wohl besten «Beweis» der Praktikabilität der von ihm propagierten Methoden. Vor diesem Hintergrund ist dieses aussergewöhnlich einprägsame Buch für jeden ärztlichen Kadermitarbeiter,

\section{KARSTEN BREDEMEIER \\ Der Rhetorik- Code}

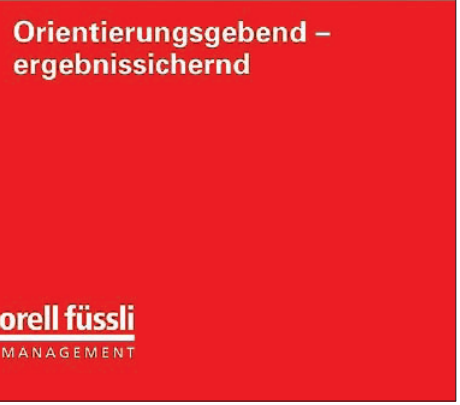

aber auch Hochschullehrer wärmstens zu empfehlen. Dem Werk ist eine weite Verbreitung $\mathrm{zu}$ wünschen, um die bestehenden Informationsdefizite im Bereich effizienter Kommunikation von (leitenden) Krankenhausärzten abbauen zu helfen.

Bernhard Schaller, Therwil 